\title{
Serum WT1-271 IgM antibody as a novel diagnostic marker for Gastric Cancer
}

\author{
MASAAKI ITO ${ }^{1}$, YUSUKE OJI ${ }^{2}$, MAYUKO ADACHI $^{2}$, RIN IMANISHI $^{2}$, SHOUQ ALZAAQI $^{2}$, \\ TAKAKI HIWASA ${ }^{1,3}$, YOKO OSHIMA ${ }^{4}$, SATOSHI YAJIMA $^{4}$, TAKASHI SUZUKI ${ }^{4}$, \\ TATSUKI NANAMI ${ }^{4}$, MAKOTO SUMAZAKI ${ }^{4}$, FUMIAKI SHIRATORI ${ }^{4}$, KIMIHIKO \\ FUNAHASHI $^{4}$, HARUO SUGIAYAMA ${ }^{5}$ and HIDEAKI SHIMADA ${ }^{1,4}$ \\ ${ }^{1}$ Department of Clinical Oncology, Toho University Graduate School of Medicine, Tokyo 143-8541; \\ ${ }^{2}$ Department of Clinical Laboratory and Biomedical Sciences, Osaka University Graduate School of Medicine, \\ Osaka 565-0871; ${ }^{3}$ Department of Neurological Surgery, Chiba University Graduate School of Medicine, Chiba 260-8670; \\ ${ }^{4}$ Department of Gastroenterological Surgery, Toho University School of Medicine, Tokyo 143-8541; ${ }^{5}$ Department of \\ Cancer Immunology, Osaka University Graduate School of Medicine, Osaka 565-0871, Japan
}

Received October 16, 2021; Accepted January 18, 2022

DOI: $10.3892 / \mathrm{mco} .2022 .2507$

\begin{abstract}
The Wilms tumor 1 gene, WT1, is overexpressed in various types of cancer, including gastric cancer. The product of WT1 is highly immunogenic and is a promising target molecule for cancer immunotherapy. The current study aimed to examine the production of WT1-specific IgG and IgM autoantibodies to identify biomarkers of diagnostic value in patients with gastric cancer. IgG antibodies that bind to WT1-derived peptides were obtained, the serum levels of which correlate with those of $\mathrm{IgG}$ antibodies against the WT1 protein in patients with intestinal malignancies. The serum levels of $\mathrm{IgG}$ and IgM antibodies against the WT1-271 peptide (271-288 amino acids) were examined in 39 healthy individuals and 97 patients with gastric cancer. The positivity cutoff value was determined according to the receiver operating characteristic curve. The association between WT1-271 IgM and the clinicopathological factors and prognosis of patients was additionally analyzed. The results revealed that serum WT1-271 IgM antibody levels in patients with gastric cancer were significantly higher than those in healthy individuals. The sensitivity and specificity of this antibody for gastric cancer were 67.0 and $71.8 \%$, respectively; this sensitivity was improved when compared with conventional tumor markers $(\mathrm{P}<0.001)$. There was no statistical difference in WT1-271 IgG antibody levels between patients with gastric cancer and healthy individuals. Serum WT1-271 IgM antibody levels were not significantly associated with clinicopathological
\end{abstract}

Correspondence to: Dr Hideaki Shimada, Department of Gastroenterological Surgery, Toho University School of Medicine, 6-11-1 Omori-Nishi, Ota-ku, Tokyo 143-8541, Japan

E-mail: hideaki.shimada@med.toho-u.ac.jp

Key words: Wilms' tumor 1, 271 antibody, IgM, gastric cancer, biomarker factors but were associated with unfavorable prognosis. Serum WT1-271 IgM antibody levels could serve as a diagnostic biomarker in patients with gastric cancer.

\section{Introduction}

A non-negligible number of patients are diagnosed in the advanced stages of gastric cancer despite advances in mass screening methods for this malignanacy. Therefore, novel biomarkers for the early detection of gastric cancer are urgently required to improve clinical outcomes. Serum autoantibodies against tumor-associated antigens (TAAs) have been reportedly found in patients with gastric cancer, including P53 (1), Muc1 (2), c-myc (3), and Survivin (4). Moreover, autoantibodies could be detected in the early stages of cancers $(5,6)$. Because of their easy accessibility and stability, autoantibodies against TAAs could serve as novel screening biomarkers.

WT1 was originally isolated as a tumor suppressor gene responsible for Wilms' tumor, a kidney neoplasm of the childhood, (7). This gene is overexpressed in various types of cancers such as leukemia (8), lung (9), colorectal (10), gastric cancer (11), and glioblastoma (12). Furthermore, its gene product is highly immunogenic (13) and proves to be a promising target molecule for cancer immunotherapy (14-17). We found that IgM and IgG antibodies against WT1 whole protein are produced in patients with hematopoietic malignancies and lung cancer (17-19). The dominant subtypes of WT1 IgG antibody were the Th1 type IgG1 and IgG3 (18) and its production requires helper T cells for IgG class switching; therefore, WT1 IgG production indicates the activation of Th1 helper $\mathrm{T}$ cells in these patients. We previously reported that the production of IgG antibody against WT1 whole protein was associated with prolonged disease-free survival in patients with tumor resected non-small cell lung cancers (19). In addition, in our therapeutic cancer vaccine trial with WT1-235 peptide for patients with recurrent glioblastoma, combining the production of WT1-235 IgG antibody and positive delayed-type hypersensitivity to the 
WT1-235 peptide was a better prognostic marker for long-term overall survival than either parameter alone (20). Associating WT1 IgG production with favorable prognosis supports the idea that IgG antibody against WT1 epitope or epitopes could act a biomarker indicating, at least, a part of WT1-specific antitumor cellular immune responses.

Compared with studies on IgG antibodies, fewer studies have analyzed the production of $\operatorname{IgM}$ antibodies in tumor-associated antigens. However, IgM antibodies are the first responders in the humoral immune system and do not depend on helper $\mathrm{T}$ lymphocytes for their production (21). Furthermore, the $\operatorname{IgM}$ receptor $\mathrm{Fc} \mu \mathrm{R}$ is also expressed on $\mathrm{T}$ and NK cells in addition to B cells in humans (22), and IgM itself may be involved in the regulation of cellular immune responses. Therefore, IgM antibodies against TAAs could be a biomarker that can indicate the antigen-specific tumor recognition of the host immune system in patients with cancer.

In the present study, we explored WT1 epitopes to identify highly antigenic epitopes in WT1 protein and identified an 18 a.a.-long WT1-271 epitope. We examined the serum positivity of WT1-271 IgG and IgM antibodies and further analyzed the association between the production of WT1-271 IgG and IgM antibodies with clinicopathological character and prognosis in 98 patients with surgically treated gastric cancer. The positivity of serum WT1-271 IgM antibody was compared with that of conventional gastric cancer tumor markers CEA and CA19-9.

\section{Materials and methods}

Collection of serum samples. This study was approved by the Ethics Committee of the Toho University Omori hospital (no. A19033) and Osaka University Hospital Ethical Committee (\#13110-10). We collected. a total of 97 serum samples from patients with gastric cancer who provided a written informed consent. All patients underwent radical surgery between January 2014 and July 2017, with 58 cases of distal gastrectomy, 5 of proximal gastrectomy, and 35 cases of total gastrectomy, at the Toho University Omori Hospital. All patients did not undergo neoadjuvant chemotherapy. The pathological stage of each patient as per the Japanese Classification of Gastric Carcinoma (23) was as follows: 52 cases with stage I, 19 with stage II, 17 with stage III, and 9 with stage IV. All patients were followed up until December 2019 or death. We examined the clinicopathological features and prognosis of gastric cancer in each patient.

Peptides. We focused on $\mathrm{IgG}$, which, at 21 days, has the longest half-life among all the isotypes of WT1 antibody. The production of IgG antibody against each WT1 peptide was analyzed against the WT1 peptide library. Next, we searched for WT1 peptide IgG antibodies that may correlate with the WT1 whole protein IgG levels. Thereafter, we analyzed the WT1 peptide antibodies that may correlate with tumor WT1 expression levels.

WT1 antigen epitopes were explored from the 18 mer-length WT1 overlapping peptide library to establish a simple enzyme-linked immunosorbent assay (ELISA) system using a WT1 peptide as a capture antigen and a tool for analyzing humoral immune responses to WT1. Notably, an 18 mer-length WT1 overlapping peptide library covering the whole
WT1 protein was synthesized at PH Japan Co. Ltd. (Hiroshima, Japan) with a purity of $>75 \%$. The amino acid sequence of the WT1-271 peptide was YESDNHTTPILCGAQYRI.

ELISA. ELISA was performed as previously reported (20) with minor modifications. The peptides $(0.2 \mu \mathrm{g} /$ well $)$ were covalently linked to a 96-well plate using the Peptide Coating kit (Takara, Shiga, Japan) as per the manufacturer's instructions. Plates were blocked using Blocking One (Nacalai Tesque, Kyoto, Japan) that had been diluted with distilled water (1:5) for $2 \mathrm{~h}$ at room temperature and washed with $0.05 \%$ TBST. The sera were diluted at 1:100 using the blocking buffer of the Peptide Coating kit. Then, $100 \mu \mathrm{l}$ diluted sera was added to each well and incubated overnight at $4^{\circ} \mathrm{C}$. After washing with Tris-buffered saline containing Tween-20 (TBST), horseradish peroxidase (HRP)-conjugated rabbit anti-human IgG (cat. no. 309-035-003; Jackson ImmunoResearch Europe, Ltd.) or HRP-conjugated goat anti-human IgM antibody (cat. no. A80-100P; Bethyl Laboratories, Inc.), diluted at 1:2,000 in TBST, was added to each well and incubated at room temperature for $2 \mathrm{~h}$. After washing with TBST, the corresponding third antibody, HRP-conjugated goat anti-rabbit IgG (cat. no. ab6721; Abcam) or HRP-conjugated rabbit anti-goat IgG antibody (cat. no. 546; MBL International Co.), diluted to $1: 5,000$ in TBST, was added to each well and incubated at room temperature for $2 \mathrm{~h}$. Bound WT1 epitope-specific IgG or IgM antibodies were colorimetrically detected using the 3,3',5,5'-tetramethylbenzidine substrate (KPL, Inc.). Absorbance was measured at $450 \mathrm{~nm}$ using a microplate reader (MULTISKAN FC; Thermo Fisher Scientific, Inc.).

Statistical analyses. Mann-Whitney U test or Chi-squared test was used to compare the unpaired groups. Fisher's exact test was used to evaluate the differences in the distribution of two variables. Kruskal-Wallis test (Mann-Whitney U test with applied Bonferroni's correction) was used to examine the corresponding differences among three variables. Logistic regression analysis was used to analyze the clinicopathological data to evaluate the association with serum WT1 antibody levels. Survival curves were calculated using the Kaplan-Meier method and compared using the log-rank test. Significant predictors were assessed using Cox proportional hazards model with multivariate analysis. EZR software (version 1.41) was used for all data analyses (24). $\mathrm{P}<0.05$ was considered to indicate a statistically significant difference.

\section{Results}

Identification of an antigenic epitope WT1-271. First, we searched for IgG antibodies that bind to WT1-derived peptides (WT1 peptide IgG antibodies) whose serum levels correlated with the serum WT1 protein IgG levels in patients with intestinal malignancies. For this purpose, we used four sera samples with high levels of WT1 protein IgG and two samples with low levels of WT1 protein IgG. Of the seven $\mathrm{IgG}$ antibodies that bound to their corresponding WT1 peptide that were identified, WT1-271 peptide (271-288 a.a.) IgG antibody was identified as a WT1 peptide IgG antibody whose serum levels may correlate with WT1 mRNA 


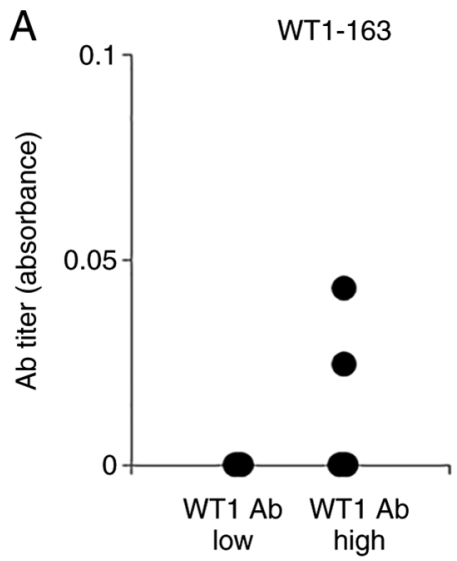

B

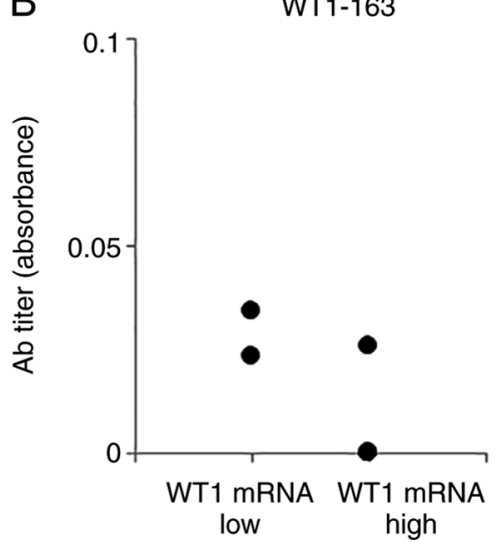

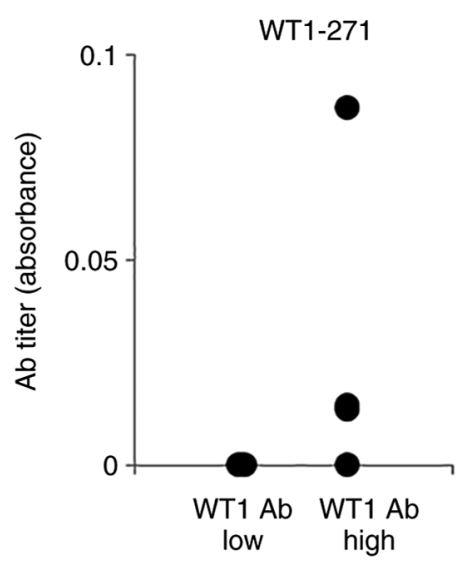

WT1-271

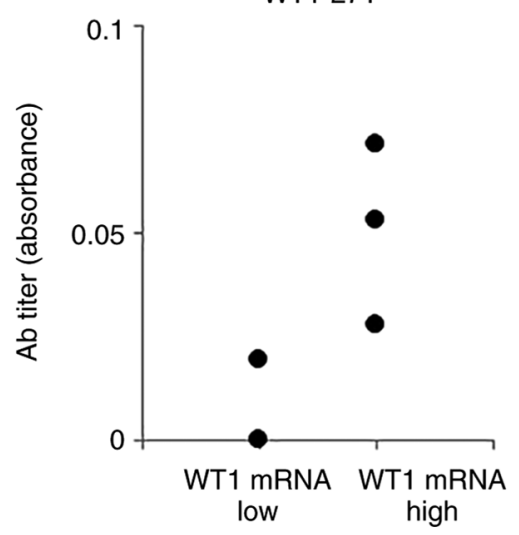

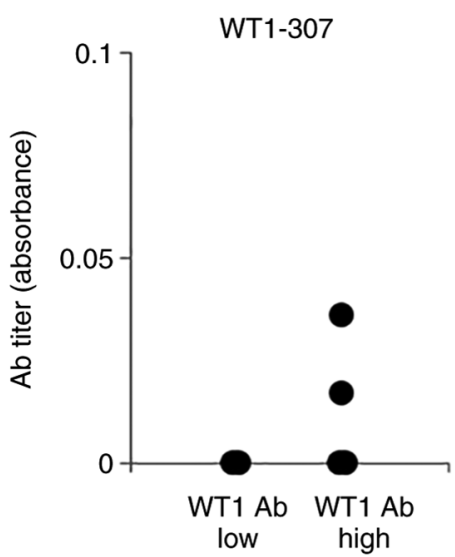

WT1-307

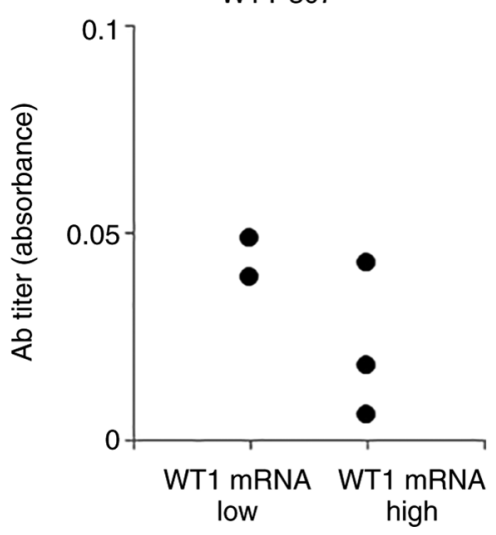

Figure 1. Identification of Wilms tumor 1-271 antibody. Serum WT1 peptide IgG antibody levels were measured by ELISA using an 18-mer length WT1 peptide. Representative results for three WT1 peptide antibodies are presented. (A) Serum WT1 peptide IgG antibody levels in patients with intestinal malignancies with high $(\mathrm{n}=4)$ and low $(\mathrm{n}=2)$ WT1 protein IgG levels. (B) Serum WT1 peptide IgG antibody levels in patients with hematological malignancies with high $(n=3)$ and low or undetectable $(n=2)$ WT1 mRNA levels in peripheral blood. Ab, antibody.

expression levels in peripheral blood using serum samples taken from five patients with hematological malignancies (Fig. 1). These results may indicate the potential of using WT1-271 IgM antibody levels as a detection marker for gastric cancer (Fig. 1).

Increased production of WT1-271 IgM antibodies in patients with gastric cancer. Production of $\operatorname{IgM}$ and $\mathrm{IgG}$ antibodies against WT1-271 epitope was investigated in 39 healthy individuals and 97 patients with gastric cancer to analyze immune recognition of the WT1 antigen by the host immune system. The median (range) antibody levels of WT1-271 IgM were 0.105 (-0.079 to 0.896$)$ for healthy individuals and 0.195 (-0.477 to 1.373$)$ for patients with gastric cancer, whereas those for $\mathrm{IgG}$ were 0.028 (-0.093 to 0.114$)$ for healthy individuals and 0.029 (-0.112 to 0.260$)$ for patients with gastric cancer. WT1-271 IgM levels in patients with gastric cancer were significantly higher than those in healthy individuals as per the Mann-Whitney $U$ test results (Fig. 2).

We performed a receiver operating characteristic (ROC) curve analysis to evaluate the sensitivity and specificity of healthy individuals and patients with gastric cancer (Fig. 3), and the results revealed that the area under the curve (AUC) value was $<0.6$ for WT1-271 IgG but $>0.6$ for WT1-271 IgM antibodies (Fig. 3A,B). When the cutoff value for IgM was considered as 0.140 , the sensitivity and specificity of serum WT1 antibody for patients with gastric cancer were 67.0 and $71.8 \%$, respectively. Next, we created a $2 \times 2$ contingency table for patients with gastric cancer and healthy individuals to present statistical evaluation. The positive predictive value was $85.5 \%$ and negative predictive value was $46.7 \%$ (Table I).

Association between serum WT1-271 IgM and IgG antibody levels with clinical stages. We focused on the pathological characteristics of 97 surgical cases of patients with gastric cancer and examined their stages (23). Stages IA and IB were integrated as I; stages IIA and IIB were integrated as II; and stages IIIA, IIIB, and IIIC were integrated as III. The mean \pm standard deviation serum WT1 IgM antibody levels for stages I $(n=52)$, II $(n=19)$, III $(n=17)$, and IV $(n=9)$ were $0.327 \pm 0.397,0.178 \pm 0.275,0.309 \pm 0.366$, and 0.353 \pm 0.308 , respectively (Fig. 4). WT1 IgG antibody levels for stages I, II, III, and IV were $0.037 \pm 0.053,0.020 \pm 0.035$, $0.049 \pm 0.057$, and $0.035 \pm 0.029$, respectively. Based on the Kruskal-Wallis test results, there was no significant association between the clinical stage and WT1-271 antibody levels. In terms of the pathological type, 57 cases were differentiated and 40 cases were poorly differentiated. Recurrence or liver, lymphatic node, bone and peritoneal metastases were observed in 19 patients. The remaining 78 patients showed no recurrence or metastasis. 
A

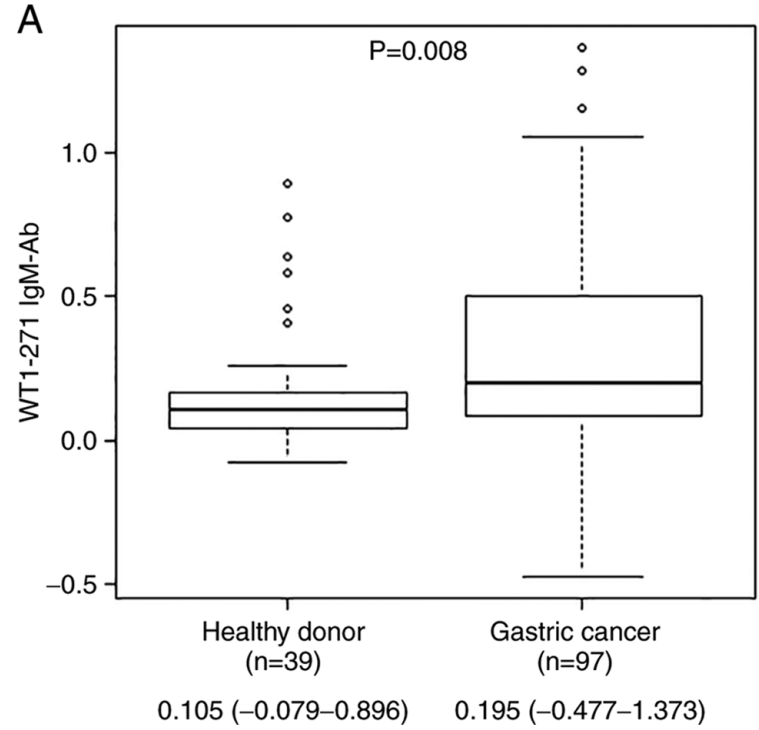

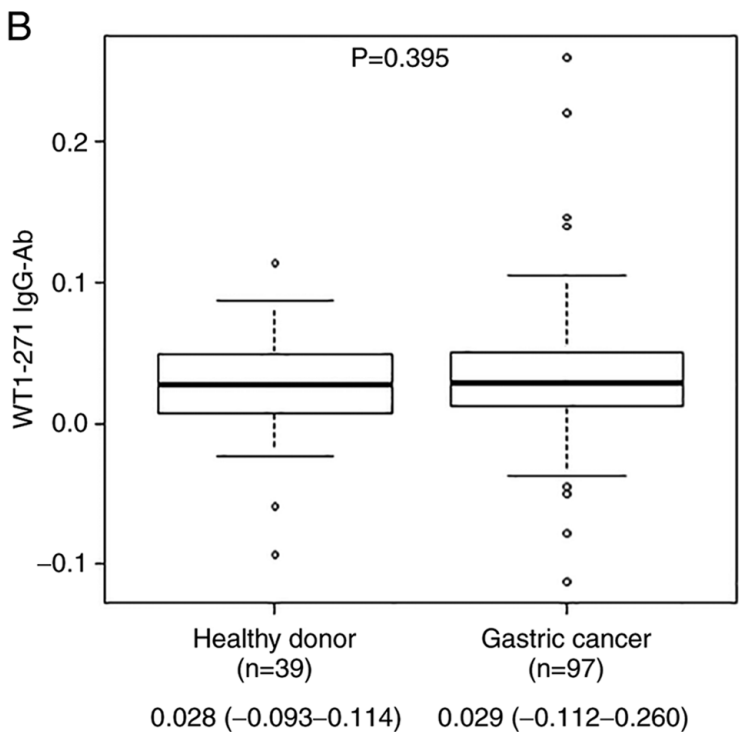

Figure 2. Comparison of serum WT1 antibody levels between healthy donors and patients with gastric cancer. s-Wilms tumor 1-271 antibody levels in healthy donors and patients with gastric cancer of (A) IgM and (B) IgG examined by ELISA. The bars and dots represent median and range of antibody level from minimum to maximum. P-values were determined using the Mann-Whitney U test. Ab, antibody.
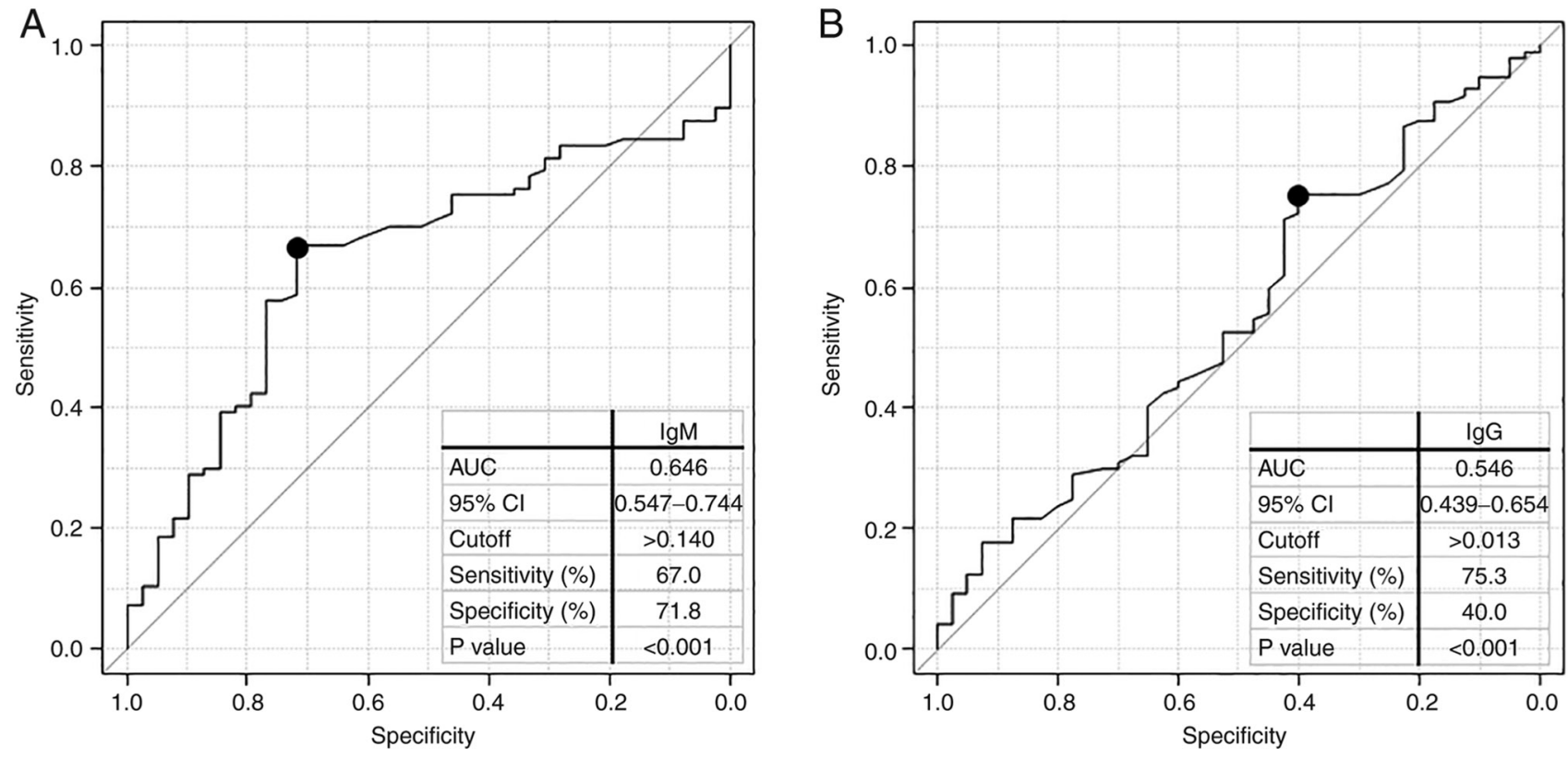

Figure 3. ROC curve of healthy donors and patients with gastric cancer. ROC curve analysis of (A) IgM and (B) IgG was performed to evaluate the sensitivity and specificity of healthy donors and patients with gastric cancer. Black dots in the figure represent the position where the sensitivity and specificity were highest. AUC, area under the curve; CI, confidence interval; ROC, receiver operating characteristic.

Association between serum WT1-271 IgM antibody levels and clinicopathological factors. We examined the association of WT1-271 IgM levels with clinicopathological factors using Fisher's exact probability test and logistic regression analysis using the cutoff level as 0.140 for the elevation of serum IgM level. According to the univariate analysis results, no significant difference was found in terms of gender, age, tumor depth (T1 vs. T2-4), lymph node (LN) metastasis and white blood cell counts, and CEA and CA19-9 levels (Table II, left panel). However, logistic regression analysis revealed that WT1-271 IgM positivity was significantly associated with earlier $\mathrm{T}$ stage ( $\mathrm{T} 1 \mathrm{vs}$. $\mathrm{T} 2-4 ; \mathrm{P}=0.049)$ but tended to be associated with advanced $\mathrm{N}$ stage ( $\mathrm{N} 0$ vs. $\mathrm{N} 1 ; \mathrm{P}=0.125)$ (Table II, right panel). Table III shows WT1-271 IgM positive rates in combination to the $\mathrm{T}$ and $\mathrm{N}$ stages. In both $\mathrm{T} 1$ and T2-4 stages, the WT1-271 IgM positive rate was higher in N1 stage than in N0.

Association between serum WT1-271 IgM antibody levels and overall survival. Using the log-rank test, we investigated the association between serum WT1-271 IgM levels and clinical outcome in patients with gastric cancer. Based on the cutoff level of 0.140 , we compared the overall survival and progression-free survival between the two groups of patients 
Table I. Correlation between WT1-271 IgM antibody positive and negative cases in healthy donors and patients with gastric cancer.

\begin{tabular}{lccc}
\hline Correlation & Gastric cancer & Healthy donor & Total cases \\
\hline Positive & 65 & 11 & 76 \\
Negative & 32 & 28 & 60 \\
\hline
\end{tabular}

The sensitivity, specificity, positive predictive value and negative predictive value were $67.0,71.8,85.5$ and $46.7 \%$, respectively. The Cutoff level was defined at 0.140 .

with high and low WT1-271 IgM levels. At 60 months after surgery, the P-values for overall survival and progression-free survival were 0.835 and 0.491 . However, similar to overall survival, progression-free survival tended to be shorter in the high WT1-271 IgM group for up to 57 months. No significant difference was found between the two groups (Fig. 5A,B). However, analysis at 6 months after surgical resection revealed that although the difference was not significant, the immunological status represented by higher serum WT1-271 IgM levels might have an unfavorable impact on the clinical outcome (Fig. S1).

Univariate analysis revealed that tumor depth, LN metastasis, and CEA and CA19-9 levels indicated significantly worse overall survival on the log-rank test. However, WT1-271 IgM levels was not significantly associated with overall survival of patients with gastric cancer (Table IV, left panel).

Multivariate analysis indicated that tumor, depth, and CEA levels were independent prognostic factors in the Cox proportional hazard model. However, no significant association was found between serum WT1 IgM levels and overall survival in patients with gastric cancer (Table IV, right panel).

WT1-271 IgM antibody levels as a detection marker for gastric cancer. We compared the detection ability of WT1-271 IgM antibodies for gastric cancer with that of the currently available tumor markers CEA and CA19-9 (Table V). In patients with gastric cancer, positive rates of CEA and CA19-9 were 9.3 and $11.3 \%$, respectively. Using chi-squared test WT1-271 IgM antibodies showed a distinctively higher positive rate of $67.0 \%$ in patients with gastric cancer $(\mathrm{P}<0.001)$. Of the 52 patients with clinical stage I disease, WT1-271 IgM antibody was detected in 35 patients (67.3\%), whereas either CEA or CA19-9 was detected in only 1 by one (1.9\%) (Table V). Even in stage II, III and IV, higher positive rate was shown in WT1-271 IgM antibodies (66.7\%) than CEA $(17.8 \%)$ or CA19-9 (22.2\%). These results may show the potential of WT1-271 IgM antibody levels as a detection marker for gastric cancer.

\section{Discussion}

In this study, we identified WT1-271, a 271-288-a.a. long WT1 sequence, as an antigenic epitope for antibody production and determined that, compared with healthy individuals, patients with gastric cancer produced significantly higher levels of WT1-271 IgM but not IgG antibodies. A cutoff level of 0.14 was determined for positive serum WT1-271 $\operatorname{IgM}$ antibodies based on the ROC analysis with an AUC of 0.6. According to this cutoff level, $67 \%$ of the patients with gastric cancer were scored as positive for WT1-271 IgM antibodies. Autoantibodies corresponded to an efficient biological amplification of the antigens in tumors and are secreted in the serum before the antigens can be detected (25). In contrast to the high positive rate of WT1-271 IgM antibodies, currently available gastric cancer tumor markers CEA and CA19-9 were positive in only 9.3 and $11.3 \%$ of the patients, respectively, in this study. This higher positivity of WT1-271 IgM antibodies is more evident in patients in clinical stage I of the disease. CEA or CA19-9 was detected in only $1.9 \%$ of the patients, whereas WT1-271 IgM antibody was detected in $67.3 \%$. Early detection is one of the most promising approaches to improve clinical outcomes for patients with cancer. Considerable efforts have been made to develop more sensitive and specific tests to detect cancer with different combinations of autoantibodies (6,26-28). Because of its high sensitivity to early gastric cancers, serum WT1-271 IgM antibody may be used as a detection marker in the screening of gastric cancer in combination with autoantibodies, especially in the early stages.

Two-thirds of the patients with gastric cancer were positive for WT1-271 IgM antibody for the cutoff level determined by the ROC analysis. Two reasons can explain this high positivity of WT1-271 IgM antibody in patients with gastric cancer. First, this can be explained by the high frequency of WT1 overexpression in gastric cancer tumors as reported in our previous immunohistochemical study, which indicated that tumor cells overexpressed WT1 protein in $42.0 \%$ of patients with gastric cancer. Moreover, WT1 overexpression was more frequent $(65.2 \%)$ in well- or moderately-differentiated gastric adenocarcinoma (11). Most patients included in the study had well- or moderately-differentiated adenocarcinomas, and thus, the majority of tumors overexpressed the WT1 protein. Second, the high positivity of the WT1-271 IgM antibody can be due to its $\mathrm{T}$ cell-independent production. In B-cell differentiation, the Ig-constant regions can be changed from IgM to other Ig isotypes through class-switch recombination. Because the class-switch into IgG is dependent on helper T cells, insufficient helper T-cell function may impair IgG production. In contrast, IgM antibodies are produced independently of helper T cells (21).

The immune system recognizes nonself in the body, and its components (immune cells and humoral factors) respond in an orchestrated manner. Production of $\operatorname{IgM}$ and IgG antibodies against the WT1-271 epitope may reflect the immune recognition and responses to the tumor-associated antigen WT1 in patients with gastric cancer. A significant increase in WT1-271 IgM in patients with gastric cancer compared with healthy individuals represents the recognition of the WT1-271 antigen by the immune system of the patient. In this study, the elevated serum WT1-271 IgM levels indicated two aspects of WT1 immune recognition in patients with gastric cancer. First, serum WT1 $271 \mathrm{IgM}$ level was increased in patients with T1N0 gastric cancer wherein the tumor remained within the gastric submucosal layer without LN metastasis. This B cell response in the very early stage of gastric cancer may support the 
Table II. Comparison of serum wilms tumor 1 IgM levels according to the clinicopathological characteristics of the patients with gastric cancer.

\begin{tabular}{|c|c|c|c|c|c|c|}
\hline \multirow[b]{2}{*}{ Variables } & \multicolumn{3}{|c|}{ Fisher's exact probability test } & \multicolumn{3}{|c|}{ Logistic regression analysis } \\
\hline & 271-IgM level $<0.140$ & 271-IgM level $\geq 0.140$ & P-value & Odds ratio & $95 \%$ CI & P-value \\
\hline Sex & & & $>0.999$ & & & \\
\hline Male & 24 & 47 & & & & \\
\hline Female & 8 & 18 & & & & \\
\hline Age & & & 0.329 & & & \\
\hline$>65$ & 21 & 50 & & & & \\
\hline$\leq 65$ & 11 & 15 & & & & \\
\hline Tumor depth & & & 0.518 & 2.994 & $0.112-0.997$ & 0.049 \\
\hline $\mathrm{T} 2-\mathrm{T} 4$ & 18 & 31 & & & & \\
\hline $\mathrm{T} 1$ & 14 & 34 & & & & \\
\hline Lymph node metastasis & & & 0.370 & 2.500 & $0.775-8.100$ & 0.125 \\
\hline $\mathrm{N} 1$ & 9 & 25 & & & & \\
\hline No & 23 & 40 & & & & \\
\hline WBC $(/ \mu 1)$ & & & $>0.999$ & & & \\
\hline$>8,000$ & 4 & 9 & & & & \\
\hline$\leq 8,000$ & 28 & 56 & & & & \\
\hline $\mathrm{CEA}(\mathrm{ng} / \mathrm{ml})$ & & & 0.472 & & & \\
\hline$>5.0$ & 4 & 5 & & & & \\
\hline$\leq 5.0$ & 28 & 60 & & & & \\
\hline CA19-9 (U/ml) & & & 0.095 & 6.520 & $0.752-56.60$ & 0.089 \\
\hline$>37.0$ & 1 & 10 & & & & \\
\hline$\leq 37.0$ & 31 & 55 & & & & \\
\hline
\end{tabular}

CA19-9, carbohydrate antigen 19-9; CEA, carcinoembryonic antigen; CI, confidence interval; N0, no lymph node metastasis; N1, lymph node metastasis exists; WBC, white blood cell.
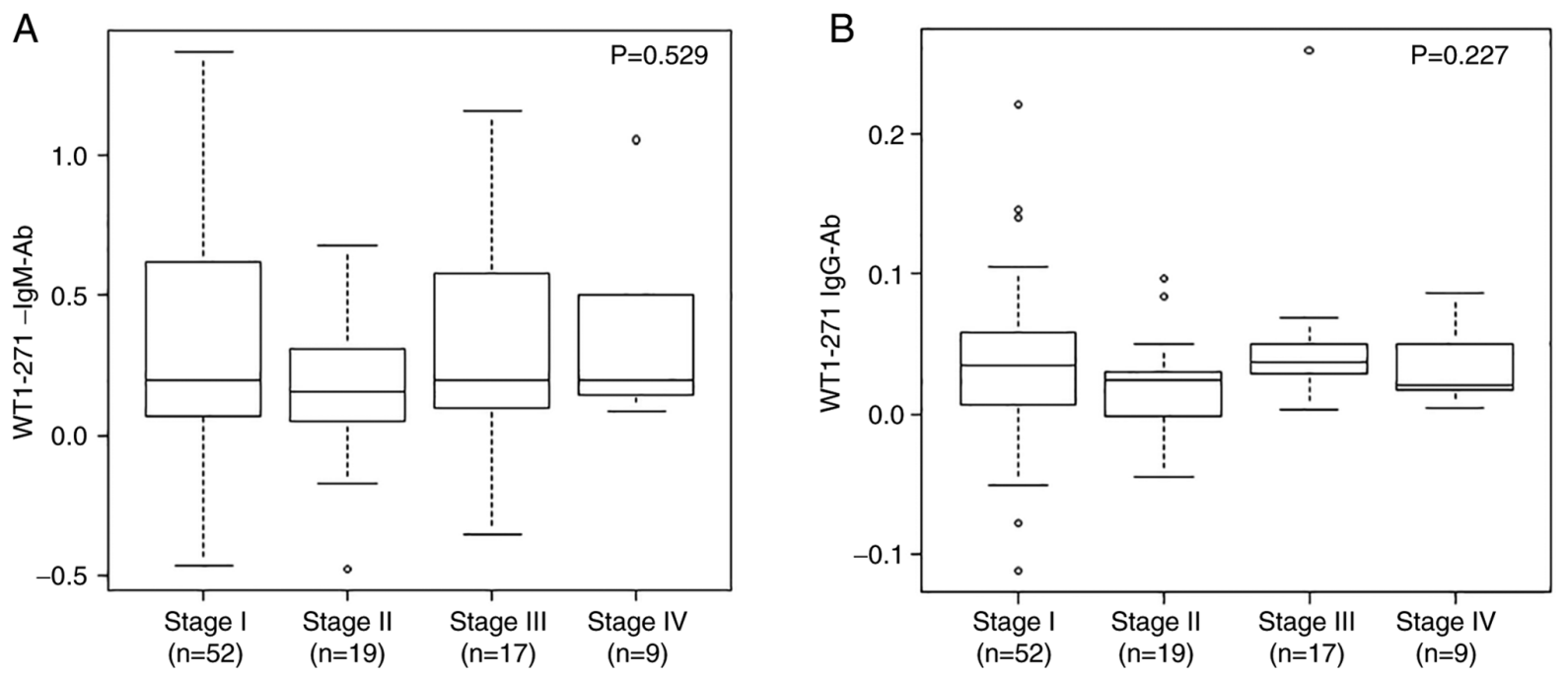

Figure 4. Comparison of serum Wilms tumor 1 antibody levels according to each stage of Japanese Gastric Cancer Association Japanese classification of gastric carcinoma. WT1-271 antibody levels of (A) IgM and (B) IgG were divided into each stage of gastric cancer. Box plot represents 25,50 and 75 percentiles. The upper and lower horizontal lines or dots represent the limits. There was no statistically significant difference between stages, as determined using the Kruskal-Wallis test. Ab, antibody.

concept of immune surveillance, which proposes eliminating cancer cells in the early stages of cancer development $(29,30)$.
Second, in T1 and T2-4 stages, the WT1-271 IgM positive rate in the N1 stage was higher than that in the N0. Reportedly, 
Table III. Wilms tumor 1-271 IgM positive rates in a combination of pathological T and N stages.

\begin{tabular}{lrrrr}
\hline Pathological stage & Negative & Positive & Total & Positive rate $(\%)$ \\
\hline T1N0 & 14 & 31 & 45 & 68.9 \\
T1N1 & 0 & 3 & 3 & 100.0 \\
T2-4N0 & 9 & 9 & 18 & 50.0 \\
T2-4N1 & 9 & 22 & 31 & 71.0 \\
\hline
\end{tabular}

N0, no lymph node metastasis; N1, lymph node metastasis exists; T1, Tumor confined to the mucosa or submucosa; T2-4, Tumor invades deeper than T1. The cutoff level was defined at 0.140 and the positive rate represents the number of positive cases/total cases x $100(\%)$.

A

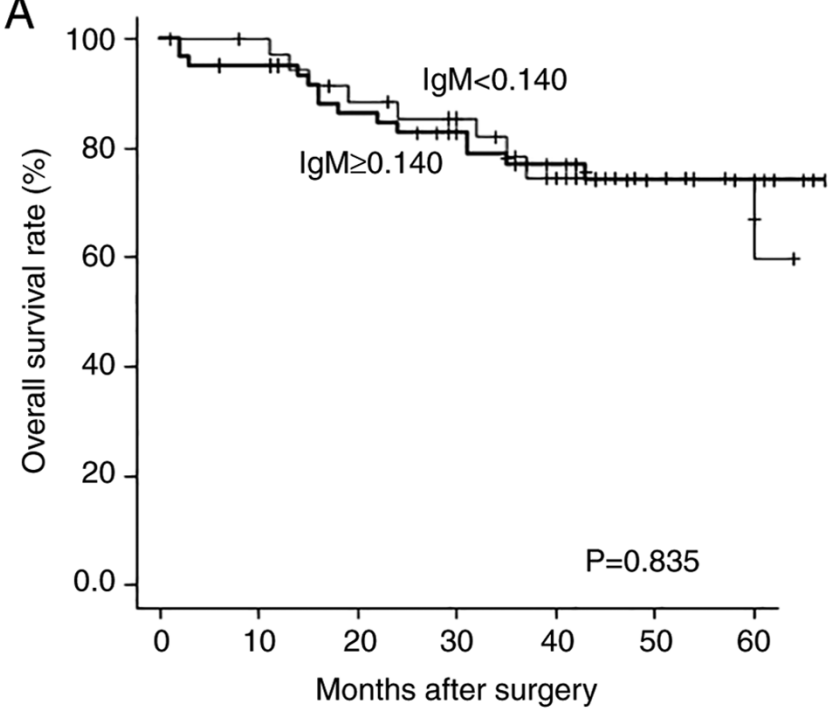

B

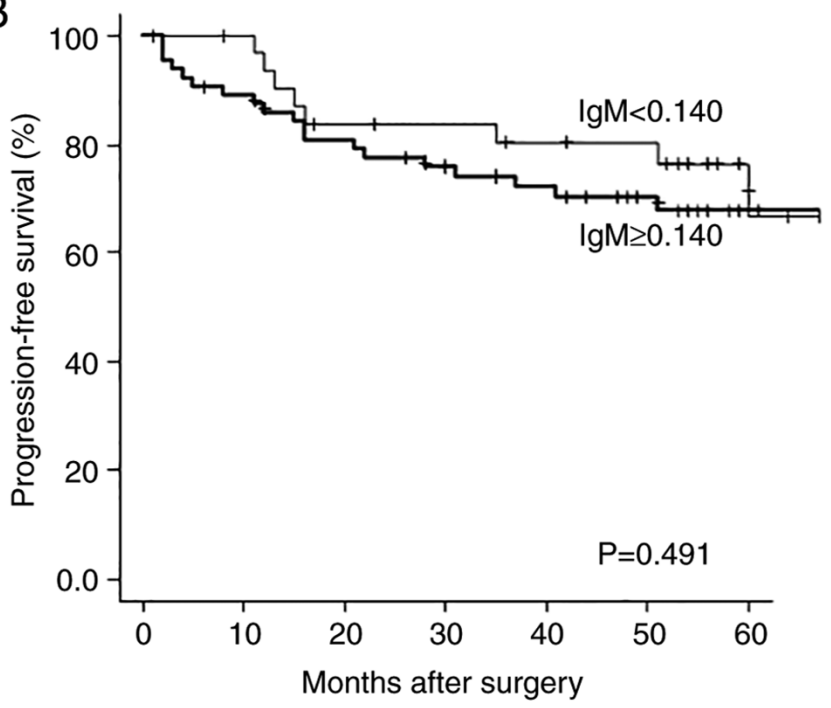

Figure 5. Serum Wilms tumor 1-271 IgM antibody levels and survival analysis. Comparison of (A) overall survival and (B) progression free survival in patients with gastric cancer according to WT1-271 antibody levels set at 0.140 by ROC curve analysis. No statistical significance was demonstrated in the two groups. The P-value at 60 months after surgery for overall survival was $\mathrm{P}=0.835$, and progression-free survival was $\mathrm{P}=0.491$. However, similar to overall survival, progression-free survival tended to be shorter in the high 271 IgM group by up to 57 months. Statistical analyses were performed using the Log-Rank test.

draining LNs are active sites for B- and T-cell responses in early-stage breast cancers. McDaniel et al (31) reported that draining LNs are a rich source of tumor-reactive B cells. In addition, Gillmore et al (32) found WT1-specific CD8 CTLs in the draining LNs in stage I/II breast cancer. These findings for breast cancer allow us to consider that the draining LNs of gastric cancer are active sites of WT1-specific immune responses, and metastasis of WT1-expressing tumor cells to the draining LNs could have triggered WT1-271 IgM production.

In the present study, we identified the WT1-271 IgG antibody as whose serum level correlated with serum WT1 protein IgG levels in patients with intestinal malignancies. It may be associated with WT1 mRNA expression levels in the peripheral blood of patients with hematological malignancies. These indicate that the WT1-271 antibodies are produced in response to an antigenic overload of WT1 antigens from tumors. Although serum WT1-271 IgM levels were elevated in most patients with gastric cancer, WT1-271 IgG levels were low and did not significantly differ from those in healthy individuals. These results indicate a lack of robust WT1-specific Th help in the patients (20). This is supported by the findings of the present study that high serum WT1-271 IgM levels is associated with unfavorable overall survival 6 months after the surgical resection (Fig. S1), although this was not statistically significant. Similar to overall survival, progression-free survival tended to be shorter in the high-WT1-271 IgM group for up to 57 months. Future studies are required to examine whether WT1-271 IgM antibody could help predict the prognosis of patients with gastric cancer.

Recently, several studies (33-36) have reported the involvement of B cells in cellular immunity as well as humoral immunity. B cells may play protumorigenic roles through the secretion of immunosuppressive cytokines, such as IL-10 $(33,34)$ and TGF- $\beta(35)$. However, B cells may also have antitumorigenic functions through IFN- $\gamma$ secretion to enhance tumor killing by NK cells and CTLs $(34,36)$ and even kill tumor cells directly via the Fas-FasL system (35). A possible association between WT1-271 IgM antibody and the unfavorable clinical outcome could partly be a result of the pro-tumorigenic cellular functions of WT1-271-specific B cells under the persistent antigenic overload of WT1 in patients with WT1-expressing gastric cancer. 
Table IV. Univariate and multivariate analysis of risk factors for the overall survival of patients with gastric cancer.

\begin{tabular}{|c|c|c|c|c|}
\hline \multirow[b]{2}{*}{ Variable } & \multirow[b]{2}{*}{ 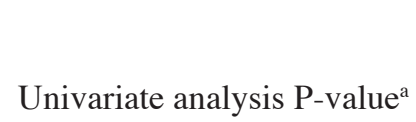 } & \multicolumn{3}{|c|}{ Multivariate analysis } \\
\hline & & Hazard ratio & $95 \% \mathrm{CI}$ & P-value ${ }^{b}$ \\
\hline Male vs. female & 0.158 & & & \\
\hline Age $>65$ vs. $\leq 65$ years & 0.068 & & & \\
\hline Tumor depth T1 vs. T2-4 & $<0.001$ & 4.945 & $1.776-13.770$ & 0.002 \\
\hline Lymph node metastasis N-vs. N+ & $<0.001$ & & & \\
\hline $\mathrm{WBC}(/ \mu 1)>8,000$ vs. $\leq 8,000$ & 0.073 & & & \\
\hline $\mathrm{CEA}(\mathrm{ng} / \mathrm{ml})>5.0$ vs. $\leq 5.0$ & $<0.001$ & 4.713 & $1.664-13.350$ & 0.004 \\
\hline CA19-9 (U/ml) >37 vs. $\leq 37$ & 0.007 & & & \\
\hline WT1 $\operatorname{IgM} \geq 0.140$ vs. $<0.140$ & 0.835 & 1.700 & $0.672-4.299$ & 0.262 \\
\hline
\end{tabular}

Table V. Relationship of WT1-271 IgM antibody positivity and each tumor marker according to gastric cancer stage.

\begin{tabular}{|c|c|c|c|}
\hline Marker & Stage I $(\mathrm{n}=52), \mathrm{n}(\%)$ & Stage II, III and IV (n=45), n (\%) & All stages $(\mathrm{n}=97), \mathrm{n}(\%)$ \\
\hline \multicolumn{4}{|l|}{$\operatorname{IgM}$} \\
\hline Negative & $17(32.7)$ & $15(33.3)$ & $32(33.0)$ \\
\hline Positive & $35(67.3)$ & $30(66.7)$ & $65(67.0)$ \\
\hline \multicolumn{4}{|l|}{ CEA } \\
\hline Negative & $51(98.1)$ & $37(82.2)$ & $88(90.7)$ \\
\hline Positive & $1(1.9)$ & $8(17.8)$ & $9(9.3)$ \\
\hline \multicolumn{4}{|l|}{ CA19-9 } \\
\hline Negative & $51(98.1)$ & $35(77.8)$ & $86(88.7)$ \\
\hline Positive & $1(1.9)$ & $10(22.2)$ & $11(11.3)$ \\
\hline All negative & $17(32.7)$ & $11(24.4)$ & $28(25.9)$ \\
\hline
\end{tabular}

IgM, wilms tumor 1-271 IgM antibody; CA19-9, carbohydrate antigen 19-9; CEA, carcinoembryonic antigen.

One-third of the healthy individuals in this study were positive for WT1-271 IgM antibodies. One explanation for this is the production of WT1-271 IgM due to the presence of latent cancer. Autoantibodies against tumor-associated antigens can appear in the early stages of cancer development and could be detected months to years before the onset of clinical symptoms. Another explanation for the high positive rate of WT1-271 IgM in healthy individuals is that the WT1-271 IgM antibody is an IgM natural autoantibody. Researchers have reported that natural autoantibodies are spontaneously produced and can exist without antigen stimulation (37-39). Historically, natural autoantibodies have been primarily associated with autoimmune diseases (37). However, compelling evidence indicates that not all natural human autoantibodies are pathogenic. Future studies are necessary to elucidate the role of both WT1-271-specific B cells and their producing WT1-271 autoantibodies in antitumor immunity of healthy individuals.
Some limitations of the study is the unknown expression of WT1 protein in the tumor cells and the lack of samples from patients with benign diseases. Future studies are warranted to demonstrate the diagnostic value of the WT1-271 IgM antibody in gastric cancer.

We identified WT1-271, a representative highly immunogenic epitope, among multiple epitopes in WT1. Preoperative serum WT1-271 IgM antibody levels in patients with gastric cancer were significantly higher than those in healthy individuals. Although the serum WT1-271 IgM antibody was not associated with clinicopathological factors, it could be used as a diagnostic biomarker for gastric cancer.

\section{Acknowledgements}

The authors would like to thank Ms. Seiko Otsuka, Ms. Chiho Kusaka and Ms. Satoko Ishibashi (Toho University Graduate School of Medicine) for preparing patient data. 


\section{Funding}

The current study was supported by the Project for Cancer Research and Therapeutic Evolution (P-CREATE) from the Japan Agency for Medical Research and Development, AMED (grant no. 21cm0106403h0006) and Grants-in-Aid for Scientific Research (KAKENHI) from the Japan Society for the Promotion of Science, JSPS (grant no. 16K10520).

\section{Availability of data and materials}

All data generated or analyzed during this study are included in this published article.

\section{Authors' contributions}

MI, YOj, HSh and HSu designed the study. MI, YOj and HSh wrote the manuscript. YOj, MA, RI and SA performed biological measurement. YOs, SY, TS, TN, MS, FS and KF collected patient sample data. MI, YOj, HSh and TH analyzed data. HSu, HSh and KF confirmed the authenticity of all the raw data. All authors read and approved the final manuscript.

\section{Ethics approval and consent to participate}

The present study was approved by the Ethics Committee of the Toho University Omori hospital, Tokyo, Japan (approval no. A19033). All patients provided written informed consent.

\section{Patient consent for publication}

Not applicable.

\section{Competing interests}

The authors declare that they have no competing interests.

\section{References}

1. Shimada H: p53 molecular approach to diagnosis and treatment of esophageal squamous cell carcinoma. Ann Gastroenterol Surg 2: 266-273, 2018.

2. Kurtenkov O, Klaamas K, Mensdorff-Pouilly S, Miljukhina L, Shljapnikova L and Chuzmarov V: Humoral immune response to MUC1 and to the Thomsen-Friedenreich (TF) glycotope in patients with gastric cancer: Relation to survival. Acta Oncol 46: 316-323, 2007.

3. Koziol JA, Zhang JY, Casiano CA, Peng XX, Shi FD, Feng AC, Chan EK and Tan EM: Recursive partitioning as an approach to selection of immune markers for tumor diagnosis. Clin Cancer Res 9: 5120-5126, 2003.

4. Megliorino R, Shi FD, Peng XX, Wang X, Chan EK, Tan EM and Zhang JY: Autoimmune response to anti-apoptotic protein survivin and its association with antibodies to p53 and c-myc in cancer detection. Cancer Detect Prev 29: 241-248, 2005.

5. Werner S, Chen H, Tao S and Brenner H: Systematic review: Serum autoantibodies in the early detection of gastric cancer. Int J Cancer 136: 2243-2252, 2015.

6. Hoshino I, Nagata M, Takiguchi N, Nabeya Y, Ikeda A, Yokoi S, Kuwajima A, Tagawa M, Matsushita K, Satoshi Y and Hideaki S: Panel of autoantibodies against multiple tumor-associated antigens for detecting gastric cancer. Cancer Sci 108: 308-315, 2017.

7. Call KM, Glaser T, Ito CY, Buckler AJ, Pelletier J, Haber DA, Rose EA, Kral A, Yeger H, Lewis WH, et al: Isolation and characterization of a zinc finger polypeptide gene at the human chromosome 11 Wilms' tumor locus. Cell 60: 509-520, 1990.
8. Inoue K, Ogawa H, Yamagami T, Soma T, Tani Y, Tatekawa T, Oji Y, Tamaki H, Kyo T, Dohy H, et al: Long-term follow-up of minimal residual disease in leukemia patients by monitoring WT1 (Wilms tumor gene) expression levels. Blood 88: 2267-2278, 1996.

9. Oji Y, Miyoshi S, Maeda H, Hayashi S, Tamaki H, Nakatsuka S, Yao M, Takahashi E, Nakano Y, Hirabayashi $\mathrm{H}$, et al: Overexpression of the Wilms' tumor gene WT1 in de novo lung cancers. Int J Cancer 100: 297-303, 2002.

10. Oji Y, Yamamoto H, Nomura M, Nakano Y, Ikeba A, Nakatsuka S, Abeno S, Kiyotoh E, Jomgeow T, Sekimoto M, et al: Overexpression of the Wilms' tumor gene WT1 in colorectal adenocarcinoma. Cancer Sci 94: 712-717, 2003.

11. Nakatsuka S, Oji Y, Horiuchi T, Kanda T, Kitagawa M, Takeuchi T, Kawano K, Kuwae Y, Yamauchi A, Okumura M, et al: Immunohistochemical detection of WT1 protein in a variety of cancer cells. Mod Pathol 19: 804-814, 2006.

12. Oji Y, Suzuki T, Nakano Y, Maruno M, Nakatsuka S, Jomgeow T, Abeno S, Tatsumi N, Yokota A, Aoyagi S, et al: Overexpression of the Wilms' tumor gene W T1 in primary astrocytic tumors. Cancer Sci 95: 822-827, 2004.

13. Cheever MA, Allison JP, Ferris AS, Finn OJ, Hastings BM, Hecht TT, Mellman I, Prindiville SA, Viner JL, Weiner LM and Matrisian LM: The prioritization of cancer antigens: A national cancer institute pilot project for the acceleration of translational research. Clin Cancer Res 15: 5323-5337, 2009.

14. Oka Y, Tsuboi A, Taguchi T, Osaki T, Kyo T, Nakajima H, Elisseeva OA, Oji Y, Kawakami M, Ikegame K, et al: Induction of WT1 (Wilms' tumor gene)-specific cytotoxic T lymphocytes by WT1 peptide vaccine and the resultant cancer regression. Proc Natl Acad Sci USA 101: 13885-13890, 2004.

15. Keilholz U, Letsch A, Busse A, Asemissen AM, Bauer S, Blau IW, Hofmann WK, Uharek L, Thiel E and Scheibenbogen C: A clinical and immunologic phase 2 trial of Wilms tumor gene product 1 (WT1) peptide vaccination in patients with AML and MDS. Blood 113: 6541-6548, 2009.

16. Anguille S, Van de Velde AL, Smits EL, Van Tendeloo VF, Juliusson G, Cools N, Nijs G, Stein B, Lion E, Van Driessche A, et al: Dendritic cell vaccination as postremission treatment to prevent or delay relapse in acute myeloid leukemia. Blood 130: 1713-1721, 2017.

17. Elisseeva OA, Oka Y, Tsuboi A, Ogata K, Wu F, Kim EH, Soma T, Tamaki H, Kawakami M, Oji Y, et al: Humoral immune responses against Wilms tumor gene WT1 product in patients with hematopoietic malignancies. Blood 99: 3272-3279, 2002.

18. Wu F, Oka Y, Tsuboi A, Elisseeva OA, Ogata K, Nakajima H, Fujiki F, Masuda T, Murakami M, Yoshihara S, et al: Th1-biased humoral immune responses against Wilms tumor gene WT1 product in the patients with hematopoietic malignancies. Leukemia 19: 268-274, 2005.

19. Oji Y, Kitamura Y, Kamino E, Kitano A, Sawabata N, Inoue M, Mori M, Nakatsuka S, Sakaguchi N, Miyazaki K, et al: WT1 IgG antibody for early detection of nonsmall cell lung cancer and as its prognostic factor. Int J Cancer 125: 381-387, 2009.

20. Oji Y, Hashimoto N, Tsuboi A, Murakami Y, Iwai M, Kagawa N, Chiba Y, Izumoto S, Elisseeva O, Ichinohasama R, et al: Association of WT1 IgG antibody against WT1 peptide with prolonged survival in glioblastoma multiforme patients vaccinated with WT1 peptide. Int J Cancer 139: 1391-1401, 2016.

21. Jones K, Savulescu AF, Brombacher F and Hadebe S: Immunoglobulin $\mathbf{M}$ in Health and Diseases: How Far Have We Come and What Next? Front Immunol 11: 595535, 2020.

22. Kubagawa H, Oka S, Kubagawa Y, Torii I, Takayama E, Kang DW, Gartland GL, Bertoli LF, Mori H, Takatsu H, et al: Identity of the elusive IgMFc receptor (FcmuR) in humans. J Exp Med 206: 2779-2793, 2009.

23. Japanese Gastric Cancer Association: Japanese classification of gastric carcinoma: 3rd English edition. Gastric Cancer 14: 101-112, 2011.

24. Kanda Y: Investigation of the freely available easy-to-use software 'EZR' for medical statistics. Bone Marrow Transplant 48: 452-458, 2013

25. Desmetz C,Mange A, Maudelonde T and Solassol J: Autoantibody signatures: Progress and perspectives for early cancer detection. J Cell Mol Med 15: 2013-2024, 2011.

26. Meistere I, Werner S, Zayakin P, Silina K, Rulle U,Pismennaja A, Šantare D, Kikuste I, Isajevs S, Leja M, et al: The prevalence of cancer-associated autoantibodies in patients with gastric cancer and progressive grades of premalignant lesions. Cancer Epidemiol Biomarkers Prev 26: 1564-1574, 2017. 
27. Yang Q, Qin J, Sun G, Qiu C, Jiang D, Ye H, Wang X, Dai L, Zhu J, Wang P and Zhang J: Discovery and validation of serum autoantibodies against tumor-associated antigens as biomarkers in gastric adenocarcinoma based on the focused protein arrays. Clin Transl Gastroenterol 12: e00284, 2020.

28. Zayakin P, Ancāns G, Silina K, Meistere I, Kalnina Z,Andrejeva D, Endzeliņ̌̌ E, Ivanova L, Pismennaja A, Ruskule A, et al: Tumor associated autoantibody signature for the early detection of gastric cancer. Int J Cancer 132: 137-147, 2013

29. Shankaran V, Ikeda H, Bruce AT, White JM, Swanson PE, Old LJ and Schreiber RD: IFNgamma and lymphocytes prevent primary tumour development and shape tumour immunogenicity. Nature 410: 1107-1111, 2001.

30. Schreiber RD, Old LJ and Smyth MJ: Cancer immunoediting: Integrating immunity's roles in cancer suppression and promotion. Science 331: 1565-1570, 2011.

31. McDaniel JR, Pero SC, Voss WN, Shukla GS, Sun Y, Schaetzle S, Lee CH, Horton AP, Harlow S, Gollihar J, et al: Identification of tumor-reactive B cells and systemic IgG in breast cancer based on clonal frequency in the sentinel lymph node. Cancer Immunol Immunother 67: 29-738, 2018.

32. Gillmore R, Xue SA, Holler A, Kaeda J, Hadjiminas D, Healy V, Dina R, Parry SC, Bellantuono I, Ghani Y, et al: Detection of Wilms' tumor antigen-specific CTL in tumor-draining lymph nodes of patients with early breast cancer. Clin Cancer Res 12: 34-42, 2006.
33. Sarvaria A, Madrigal JA and Saudemont A: B cell regulation in cancer and anti-tumor immunity. Cell Mol Immunol 14: 662-674, 2017.

34. Tao H, Lu L, Xia Y, Dai F, Wang Y, Bao Y, Lundy SK, Ito F, Pan Q, Zhang X, et al: Antitumor effector B cells directly kill tumor cells via the Fas/FasL pathway and are regulated by IL-10. Eur J Immunol 45: 999-1009, 2015.

35. Olkhanud PB, Damdinsuren B, Bodogai M, Gress RE, Sen R, Wejksza K, Malchinkhuu E, Wersto RP and Biragyn A: Tumor-evoked regulatory B cells promote breast cancer metastasis by converting resting CD4+ T cells to T-regulatory cells. Cancer Res 71: 3505-3515, 2011.

36. Schwartz M, Zhang Y and Rosenblatt JD: B cell regulation of the anti-tumor response and role in carcinogenesis. J Immunother Cancer 4: 40, 2016.

37. Fereidan-Esfahani M, Nayfeh T, Warrington A, Howe CL and Rodriguez M: IgM Natural autoantibodies in physiology and the treatment of disease. Methods Mol Biol 1904: 53-81, 2019.

38. Meffre E and Salmon JE: Autoantibody selection and production in early human life. J Clin Invest 117: 598-601, 2007.

39. Elkon K and Casali P: Nature and functions of autoantibodies. Nat Clin Pract Rheumatol 4: 491-498, 2008. 